\title{
Imaging the Morphology of Solvent-Free Prepared MALDI Samples
}

\author{
Scott D. Hanton, Todd M. McEvoy, and James R. Stets \\ Air Products and Chemicals, Inc., Allentown, Pennsylvania, USA
}

Matrix-assisted laser desorption/ionization (MALDI) mass spectrometry is an important technique to characterize many different materials, including synthetic polymers. MALDI mass spectral data can be used to determine the polymer average molecular weights, repeat units, and end groups. The development of solvent-free sample preparation methods has enabled MALDI to analyze insoluble materials and, interestingly, can provide higher-quality mass spectral data. Although the utility of solvent-free sample preparation for MALDI has been demonstrated, the reasons for its success are only now being discovered. In this study, we use microscopy tools to image samples prepared using solvent-free methods to examine the morphology of these samples. The samples are prepared using a simple vortex method. Our results show that the average particle size of typical MALDI matrices is reduced from their original tens to hundreds of micrometers to hundreds of nanometers. This size reduction of the matrix occurs in one minute using the vortex method. We also observe remarkably smooth and homogeneous sample morphologies for the laser to interrogate, especially considering the relatively crude methods used to prepare our samples. (J Am Soc Mass Spectrom 2008, 19, 874-881) (C) 2008 American Society for Mass Spectrometry

$\mathrm{M}$ atrix-assisted laser desorption/ionization (MALDI) mass spectrometry [1-4] has become an important technique to characterize the chemical structure of many different analytes, including industrial polymer materials [5-10]. MALDI can generate important data on the telomer repeat units, end groups, and average molecular weights of these materials. MALDI methods have been developed to address a broad variety of different polymer materials containing different chemistries. One of the key issues in traditional MALDI sample preparation is making true solutions of the analyte and the matrix [9]. Many interesting polymeric analytes are either completely insoluble or present significant challenges in making analytically useful solutions. To address these issues, solvent-free sample preparation methods have been developed. While several groups investigated solventfree sample preparation methods at nearly the same time, the methods developed by Trimpin, Räder, and coworkers have gained widespread use [11-15]. To make the sample preparation step easier, less time consuming, and reduce the risk of cross-contamination, we developed a simple version of the solvent-free sample preparation method, now called the vortex method [16]. Although the utility of solvent-free sample preparation for MALDI has been clearly demonstrated, the investigations into why it works and what impact

Address reprint requests to Dr. Scott D. Hanton, Air Products and Chemicals, Inc., GAS, 7201 Hamilton Blvd., Allentown, PA 18195. E-mail: hantonsd@airproducts.com these data have on our overall understanding of the MALDI process have only recently begun [17, 18].

To develop increased understanding of the utility of the solvent-free MALDI sample preparation method, we have investigated the morphology of these samples using different imaging experiments-optical imaging [19], scanning electron microscopy (SEM) [20], atomic force microscopy (AFM) [21], and time-of-flight secondary ion mass spectrometry (ToF-SIMS) [22]. Previous work has shown the utility of investigating MALDI sample morphology using imaging methods. We have used SEM to investigate the morphology of electrospray deposited samples [23] and used ToF-SIMS imaging to compare air dry, electrospray, and multiple-layer deposited samples [24, 25]. The previous ToF-SIMS experiments demonstrated that increased homogeneous morphology correlated to improved MALDI success. We understand this to be the presence of many different matrix-analyte interactions within the volume of the focused laser on the MALDI target. By using these three different imaging techniques, we can obtain highresolution area images using SEM, high-resolution height images using AFM, and ion-specific images using ToF-SIMS.

In this report, we will show that microscopy imaging experiments demonstrate a high degree of mixing in our relatively simple vortex mixing experiments that for the size scale of the focused laser beam in a MALDI experiment, generate remarkably homogeneous samples. We will also show that matrices undergo interesting changes in morphology during vortex mixing, 
and that different matrices show very different local morphology.

\section{Experimental}

\section{Chemicals}

The polyethylene glycol (PEG) samples were obtained from Aldrich Chemical (Milwaukee, WI, USA). The polymethylmethacrylate (PMMA) and polystyrene (PS) samples were obtained from American Polymer Standards (Mentor, $\mathrm{OH}, \mathrm{USA}$ ). The polyethynl formamide (PEF) samples were produced at Air Products and Chemicals (Allentown, PA, USA) as research materials. All of the polymer analytes are relatively low average molecular mass, with number-average molecular weights $\left(\mathrm{M}_{\mathrm{N}}\right)$ ranging from about 1000 to $5000 \mathrm{Da}$. The samples were prepared for MALDI using the following matrices: 2,5-dihydroxybenzoic acid (DHB), $\alpha$-cyano-4hydroxycinnamic acid (CHCA), ferulic acid (FA), indole acrylic acid (IAA), dithranol, and diphenyl butadiene (DPBD), all of which were obtained from Aldrich. All of the matrices were of a standard grade. No high-purity grades were used or additional purification done. Samples doped with a specific cationization agent used sodium trifluoroacetate (NaTFA) or silver trifluoroacetate (AgTFA), both obtained from Aldrich. All chemicals were used as received.

\section{Sample Preparation: Vortex Method}

The vortex method [16] involves adding the dry components of the analyte (about 0.1-0.2 mg), matrix (20-40 mg), and cationization agent (trace to $0.1 \mathrm{mg}$ ) to a small glass vial. We typically add the analyte first, followed by the matrix and then the cationization agent, but there is no clear reason why the order of addition would matter to these experiments. Two BBs (premium grade, $4.5 \mathrm{~mm} \mathrm{Zn}$ plated, ultra smooth, steel air gun shot from Daisy Outdoor Products, Rogers, AR, USA) are then added to the vial. The contents in the vial are mixed on a vortex mixer for $60 \mathrm{~s}$. A small spatula is used to remove a few grains of the mixed sample from the vial and transfer a few grains to the MALDI target. Using the flat edge of the spatula, and moderate pressure, we flatten the grains of material on the target in a circular pattern. This process requires only a few rotations and creates a thin film about $2-3 \mathrm{~mm}$ in diameter. Once a thin film is observed on the target, sufficient material is present for the MALDI experiment. Any extra material is removed from the target by gently tapping the target on the benchtop. Apparently the majority of the material originally transferred to the target is removed during tapping. A minority of the material forms the thin film for further analysis.

\section{Mass Spectrometry}

All of the MALDI experiments were conducted on a Bruker Biflex III (Billerica, MA, USA) TOF mass spectrometer. These experiments were all conducted in reflectron mode using delayed extraction. The Biflex was equipped with a nitrogen laser operated at $337 \mathrm{~nm}$ and $3 \mathrm{~Hz}$. The laser fluence was optimized to be slightly above threshold for ions. The laser fluence was optimized differently for each matrix molecule used depending on their relative absorptivities. The source conditions of the mass spectrometer were optimized for mass resolution near the peak of the polymer telomer distribution. Spectra were collected by moving the sample target relative to the laser and averaging 125 shots/spectrum. The data were analyzed using Polymerix software (Sierra Analytics, Modesto, CA, USA).

\section{Imaging}

The optical imaging experiments were conducted on a Nikon SMZ-U Stereoscope equipped with a Nikon Digital Sight Camera system, which includes a Nikon DS-5M camera and Nikon DS-U1 controller unit. The image forming portion of the digital camera (e.g., the CCD) is an array of $2560 \times 1920$ pixel elements, equivalent to 5 megapixels, each of which is $3.4 \times 3.4$ $\mu \mathrm{m}$ in size. The camera captures images in a 12-bit image depth providing a high level of color density $\left(2^{12}=4096\right.$ color shades). Image acquisition and processing were performed using EclipseNet (Nikon).

The SEM experiments were conducted on a JEOL JSM-5910LV scanning electron microscope operated in low-vacuum mode with a pressure in the specimen chamber of about 20 Pascals. No conductive metal coating was applied to the specimens. The accelerating voltage was $20 \mathrm{kV}$. All images were collected using the backscattered electron signal.

Atomic force microscopy experiments were performed using a Digital Instruments Dimension 3000 (Veeco Metrology, Santa Barbara, CA, USA) equipped with a Nanoscope IIIa controller. All measurements were obtained in tapping mode with springboard style etched silicon (RTESP) Nanoprobe SPM tips (Veeco Metrology, cantilever length $125 \mu \mathrm{m}$ and resonance frequency of about $350 \mathrm{kHz}$ ). All images were acquired at scan rates of $0.4-0.5 \mathrm{~Hz}$ and line densities of $512 \times$ 512 pixels.

ToF-SIMS experiments were conducted on a Physical Electronics (Eden Prairie, MN, USA) TRIFT ${ }^{\circledR}$ II time-offlight mass spectrometer equipped with a ${ }^{69} \mathrm{Ga}$ liquidmetal ion gun. The instrument measures mass via time-of-flight, but incorporates both a short linear flight tube and three electrostatic sectors for a curved flight path. The total flight path is about $2 \mathrm{~m}$. Ions are detected by a dual microchannel plate (MCP) detector. Only positive ion mass spectra are reported herein.

For ToF-SIMS experiments, a 600-pA, 25-kV (20-ns pulse width, 250-nm probe size) unbunched primary 
ion beam for high lateral resolution ion imaging were typically used. The lateral resolution is both sample and secondary-ion yield dependent, but for our experiments at $25 \mathrm{kV}$ was typically about $1-2 \mu \mathrm{m}$. Typical repetition rates were $5-15 \mathrm{kHz}$. Total primary-ion doses were less than $10^{-12}$ ions $/ \mathrm{cm}^{2}$. The raster areas varied from $50 \times$ $50 \mu \mathrm{m}$ for high-resolution imaging to $400 \times 400 \mu \mathrm{m}$. The secondary ions were extracted using an electric field imparting $3.2 \mathrm{keV}$ of kinetic energy. Ions experienced $7 \mathrm{kV}$ of post-acceleration just before detection. The MCP detector was held at $1350 \mathrm{~V}$. Signals from the detector were processed by a multistop time-to-digital converter with 138-ps time resolution. Data acquisitions averaged between 10 and $30 \mathrm{~min}$.

In static SIMS experiments of insulating samples, surface charging is often a problem. The TRIFT instrument is equipped with a pulsed charge compensator to mitigate this problem. Unlike previous ToF-SIMS experiments of MALDI samples, where no charging issues were observed while examining air dry and electrospray deposited samples [24], significant problems with surface charging were sometimes encountered. All of these experiments were conducted with charge compensation. The charging problems were surprising and indicate that, despite the relatively crude methods used to create the thin films to be analyzed by MALDI, this sample preparation method generated substantially complete films with little or no leakage through or around the films. No correlation was discovered between analytes, matrices, or cationization agents between samples that showed moderate charging problems, or samples that showed substantial charging problems. The charging issues experienced in these samples also indicate that the lack of charging experienced in the electrospray deposited samples demonstrates that those films have significant pathways through the film to the metal target [24].

\section{Results and Discussion}

The matrices used in typical MALDI experiments come from the vendors as rather coarse, solid materials. We observe significant reduction in the particle size of the matrix during the vortex mixing. Figure 1 shows optical images at a 5-fold magnification of the as-received
CHCA matrix (left) and a sample of PS 2450 prepared with CHCA and AgTFA (right). The as-received CHCA matrix particles are on average $500 \mu \mathrm{m}$ in diameter. Vortex mixing with subsequent application to the target results in the formation of particle aggregates which are about $50 \mu \mathrm{m}$ in diameter. By optical microscopy and AFM (see following text; see also Figure 4, below) we observe that these are truly particle aggregates, which consist of even smaller individual particles. These images demonstrate a substantial reduction in the typical particle size of the CHCA matrix particles with only $60 \mathrm{~s}$ of vortex mixing, followed by application to the MALDI target.

Figure 2 shows the resulting MALDI mass spectrum for the sample shown in Figure 1b. The y-axis is ion intensity. The simple vortex method enables highquality MALDI mass spectra of the polymers in this study. All of the samples for which images are shown herein produced excellent MALDI mass spectra, similar in quality to Figure 2. Since these analytes are generic, low mass polymers, which have been exhaustively analyzed, further mass spectra are not shown. No significant information was obtained from the various mass spectra; moreover, the sample preparation methods were all successful for MALDI.

Although the typical particle size is greatly reduced, either the vortex mixing or the spatula application could be responsible for the reduction in matrix particle size. To measure the relative impact of each, we obtained SEM images of raw matrix, the sample from the vortex mixer, and the sample as applied on a MALDI target. Figure 3 shows the results for a sample of PMMA 2k prepared with IAA. The top image (Figure 3a) shows the SEM of the raw IAA, as received. We see large rectangular crystals of IAA. Analyzing the image in Figure 3a, we observe a typical as-received rectangular crystal size of about 50-100 $\mu \mathrm{m}$. The middle image (Figure $3 b$ ) shows a substantial change in the morphology of the sample after vortex mixing for $60 \mathrm{~s}$. The sample now shows primarily round particles with a typical particle size of $4-10 \mu \mathrm{m}$ (diameter). The bottom image (Figure 3c) shows the same sample after application to a MALDI target using the metal spatula with moderate pressure. The white areas observed in Figure
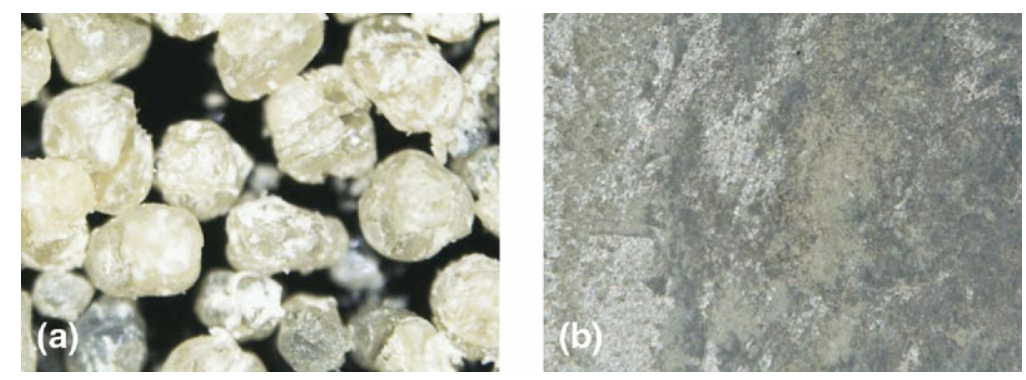

Figure 1. Optical microscopy with $5 \times$ magnification of the raw CHCA matrix (a, left) and the sample prepared using the vortex method and applied to an $\mathrm{Al}$ substrate (b, right). The images shown are $1.9 \times 2.5 \mathrm{~mm}$. 


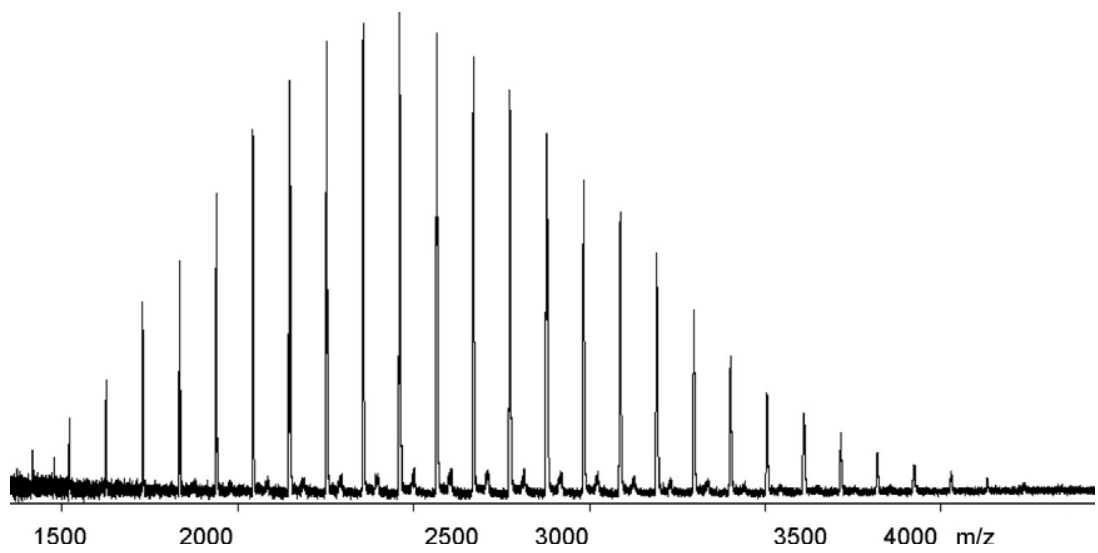

Figure 2. MALDI mass spectrum of PS 2450 prepared solvent-free with CHCA as the matrix and AgTFA as the cationization agent. This figure is typical of the MALDI data obtained from all of the polymer samples prepared in this study. Since all of the polymer analytes are generic, low-mass polymers, no significant information was obtained from the various mass spectra, besides that the sample preparation methods were all successful for MALDI.

3c are the Al substrate upon which the sample was prepared.

The middle (b) and bottom (c) frames of Figure 3 show essentially similar images. The typical particle size in each image is about $4-10 \mu \mathrm{m}$. It is clear from these data that the vortex mixing is primarily responsible for the reduction in average particle size of the matrix, and for the change in the shape of the crystals from rectangular to round. This experiment indicates that the application of the sample to the target with the spatula has little impact on the final particle size observed in the samples. The spatula is simply transferring the particles generated in the vortex mixing to the target for analysis. Perhaps the most interesting point is that this reduction in particle size was accomplished in only $60 \mathrm{~s}$ of vortex mixing.

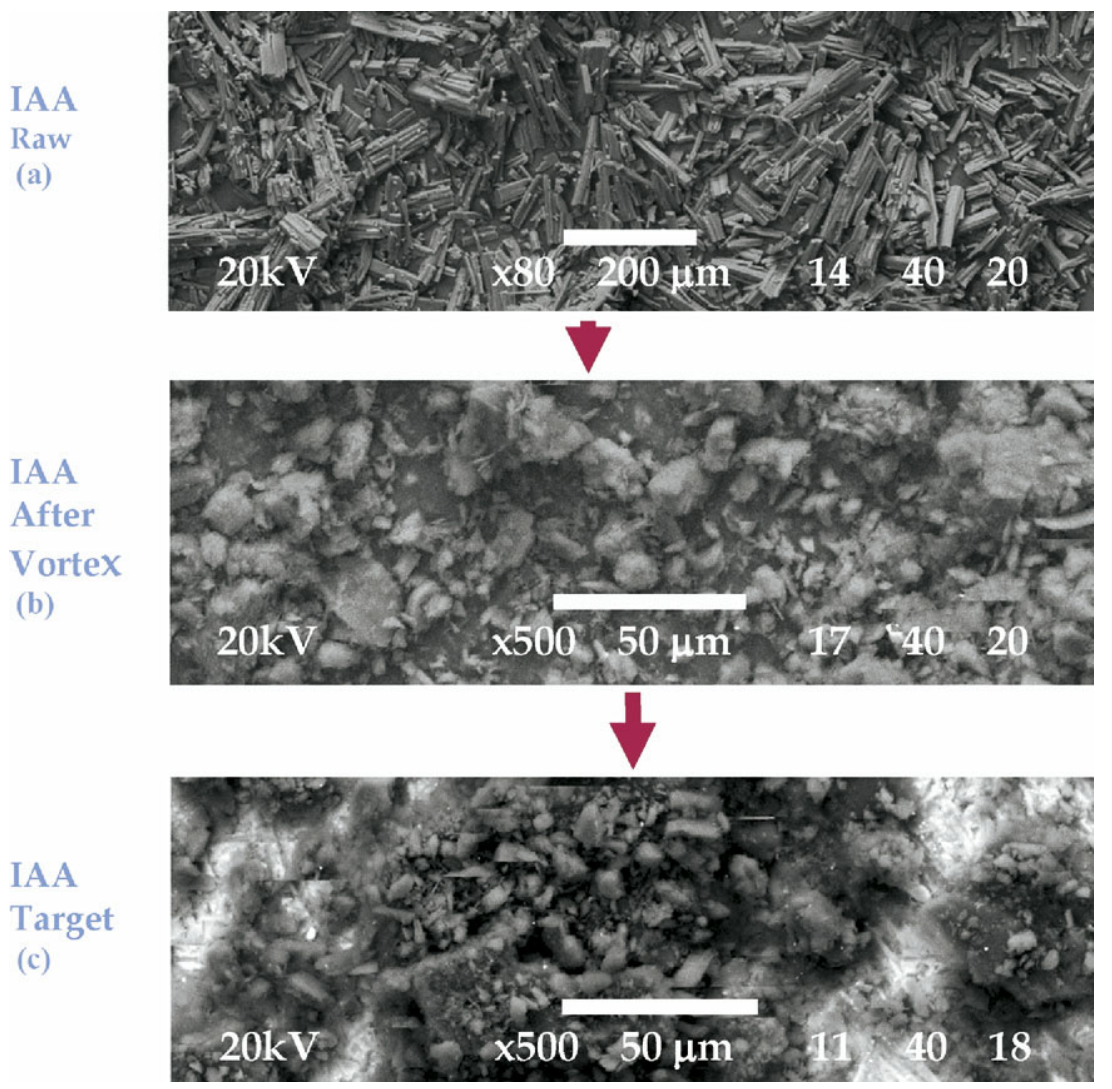

Figure 3. SEM images of samples of PMMA $2 \mathrm{k}$ prepared with IAA: raw crystals, as received (a, top), sample after vortex mixing (b, middle), sample after spatula application to the substrate (c, bottom). 

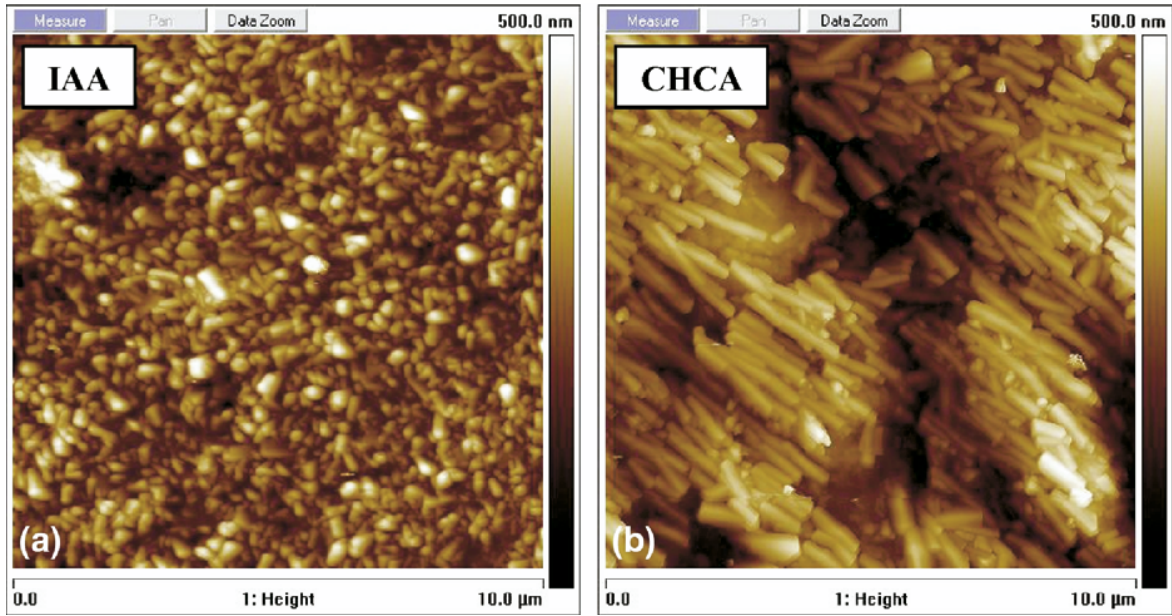

Figure 4. AFM images $(10 \times 10 \mu \mathrm{m})$ of samples of PMMA $2 \mathrm{k}$ prepared with IAA $(\mathbf{a}$, left $)$ and CHCA (b, right) as the matrix. The images show that only $60 \mathrm{~s}$ of vortex mixing changes the typical particle size of the matrix, and creates a surprisingly homogeneous sample. The vertical scale for both images is $500 \mathrm{~nm}$.

The change in the IAA local particle shape from generally rectangular to generally round was very interesting. Using AFM microscopy we investigated the local shape of different matrices after vortex mixing. Figure 4 shows AFM images of samples of PMMA 2k prepared using IAA and CHCA. As observed in the SEM results, the IAA sample (Figure 4a) clearly has a round shape after vortex mixing. Comparing the CHCA image (Figure $4 \mathrm{~b}$ ) to the optical image in Figure 1, we see another change in the local shape of the matrix crystals. The CHCA crystals have changed from very large spherical crystals to much smaller rod-shaped crystals. The reason for these changes is not yet clear. The local shape observed is due to the matrix. We observe this shape change independent of which polymer analyte is prepared with the matrix [26]. This indicates that the shape change results from physical deformation of the matrix and not the specific polymer being analyzed.

Another feature of the AFM images shown in Figure 4 is the high degree of homogeneity of the samples. Although there is a slight particle size distribution for each sample analyzed with AFM, the images show a rather consistent final particle size for each matrix. In Figure $4 a$, the particles from the sample prepared with IAA have a typical diameter of about $250 \mathrm{~nm}$. In Figure $4 \mathrm{~b}$, the particles from the sample prepared with CHCA result in elongate particles that exhibit widths of about $250 \mathrm{~nm}$ and lengths of about $1 \mu \mathrm{m}$. If we compare these sizes to the as-received matrix crystals analyzed in Figure 1a (CHCA) and Figure 3a (IAA), we observe a significant reduction in typical size. The CHCA particles are reduced in size from about $500 \mu \mathrm{m}$ in diameter to elongates that are typically $250 \mathrm{~nm}$ by about $1 \mu \mathrm{m}$. The IAA particles are reduced in size from elongates that are typically about $70 \times 10 \mu \mathrm{m}$ to spheres that are typically $250 \mathrm{~nm}$ in diameter. By volume, an average CHCA particle has been reduced by a factor of about a billion
[27]. By volume the average IAA particles have been reduced by a factor of about a million.

Although the shapes are different for samples prepared with IAA and CHCA, the final particle size is surprisingly consistent for such a simple method as the vortex method. Previous work has shown a correlation between highly homogeneous MALDI sample morphology and increased reproducibility of the MALDI mass spectra obtained [23], whereas we define the critical size parameter for homogeneous behavior to be about the size of the laser spot on the target during the MALDI experiment. Apparently part of the explanation of the success of the solvent-free sample preparation is the homogeneous samples that result.

During the AFM experiments, we were unable to obtain images of the samples prepared with dithranol as the matrix. The samples made with the dithranol matrix were simply too rough for measurement by AFM [28]. The SEM image of a sample of PS prepared with dithranol and AgTFA shown in Figure 5 demonstrates why. In Figure 5, we observe two fundamentally different morphologies, a flat, mostly homogeneous plain, strewn with large boulders. Although some of the largest boulders observed in Figure 5 can be identified as conglomerates of smaller particles, the typical particle size observed for these large particles ranges from 3 to $10 \mu \mathrm{m}$. The particles comprising the plain are more difficult to characterize. Unfortunately, the boulders were too tall to obtain useful AFM images from the plain. Even with $500 \times$ magnification in Figure 5, the plain appears homogeneous and featureless. Figure 6 shows a higher-magnification image of the same sample taken with $2000 \times$ magnification in an area of the smooth plain. Even at $2000 \times$ magnification, these areas look smooth and contiguous. It is difficult to quantitatively measure a typical particle size, but these data indicate a particle size after vortex mixing of $<1 \mu \mathrm{m}$. 


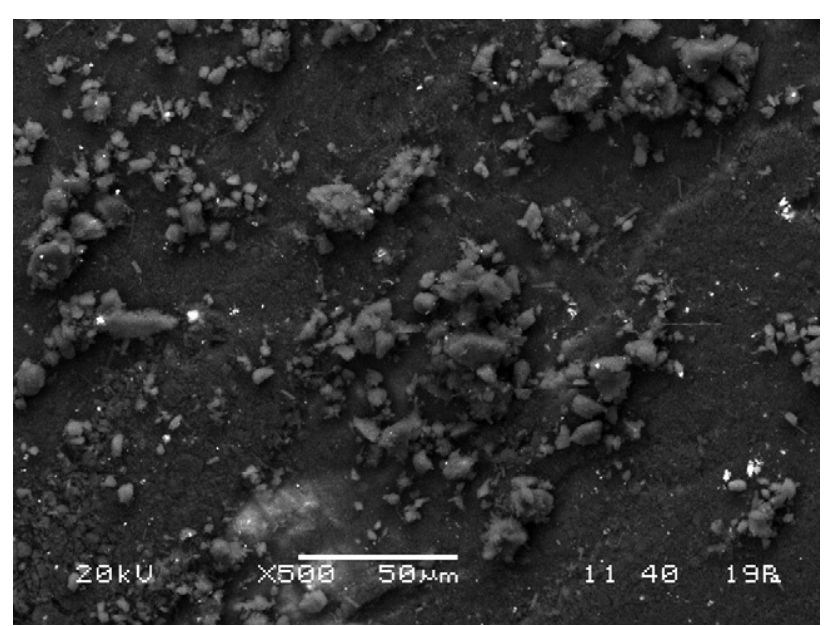

Figure 5. SEM image of a sample of PS 2450 prepared with dithranol and AgTFA. The image shows two very different morphologies, a very smooth and homogeneous plain strewn with large boulders. Subsequent SIMS analysis shows that excellent polymer mass spectra are obtained only from the flat regions. The boulders are assigned as dithranol particles.

To further investigate the dithranol sample morphology, we obtained ToF-SIMS images of the same sample investigated in Figures 5 and 6. Figures 7 and 8 show ion-specific SIMS images of the sample of PS 2450 prepared with dithranol and AgTFA at different lateral image resolutions. Figure 7 had a raster area of $250 \times$ $250 \mu \mathrm{m}$, whereas Figure 8 had a raster area of $50 \times 50$ $\mu \mathrm{m}$. The ion images shown include the total ion (all observed ions), dithranol, Ag, Na (Figure 7 only), $\mathrm{Al}$, and two ion images relating to the PS analyte, intact telomers, and the typical PS fragment observed at 91 Da. The figures are plotted with a thermal scale showing relative ion intensity. To ease the interpretation of these figures red $<$ yellow $<$ white.

As mentioned earlier in the experimental section on the SIMS images, charging was a significant problem in many of the samples analyzed. This sample (PS 2450 prepared with dithranol and AgTFA, Figures 7 and 8) was one of the most difficult samples from which to acquire high-quality SIMS images. This sample (and many others) were highly insulating in the SIMS and SEM experiments. The thin film plain observed in Figures 5 and 6 is quite complete. There are few flaws in the film that enable the metal substrate to drain excess electrons and dissipate the charge building up on the film.

A comparison of the total ion image and the specific ion images for dithranol and Ag ions in Figure 7 shows that the large features observed in the total ion image are composed primarily of dithranol and the $\mathrm{Ag}$ salt. The only trace of the large features observed in the total ion image found in the other ion specific images is the hole in the PS 91 Da fragment ion image. Apparently the matrix boulder has covered the PS material, and no $91 \mathrm{Da}$ ions were observed in that part of the image. For this matrix, the vortex mixing does not appear to grind all of the matrix to small typical particle size as efficiently as with the other matrices studied. In addition, during the collection of the SIMS mass spectral data, we observed that usable mass spectra of the PS telomers could be obtained only from the featureless, smooth areas of the sample. The areas that showed distinct features, or the boulders observed by SEM, did not produce the PS telomer distributions.

In addition to the boulders observed in Figure 7, Figures 7 and 8 show largely homogeneous regions of the components of the sample. We cannot distinguish individual particles in these images, so the average particle size must be less than about $1 \mu \mathrm{m}$. In this smooth, homogeneous plain, the dithranol, PS, and Ag ions are rather evenly distributed. These images show essentially the same behavior as previously reported for electrospray deposition samples [23]. In both deposition methods we observe evenly distributed matrix, analyte, and salt in the SIMS images. This even distribution of the key sample components may be an important part of the reason why the vortex method can produce such high-quality mass spectra. In practice, during the MALDI experiment, the necessary components of the experiment (analyte, matrix, and cationization agent) are all plentiful and available within the area of the laser spot on the target. The absence of solvent may aid these experiments by eliminating the crystallization effects that impart heterogeneous morphology to some solventbased MALDI samples [14]. Now that we observe the high degree of homogeneity in these samples, the key question is how $60 \mathrm{~s}$ of vortexing and a simple spatula transfer can create such a homogeneous thin film for the MALDI experiment to interrogate?

To examine the quality of the thin films, we also mapped the images of the $\mathrm{Al}^{+}$ion, from the aluminum MALDI target. In both Figures 7 and 8 we see a low signal level, homogeneous image for $\mathrm{Al}^{+}$. Initially, we

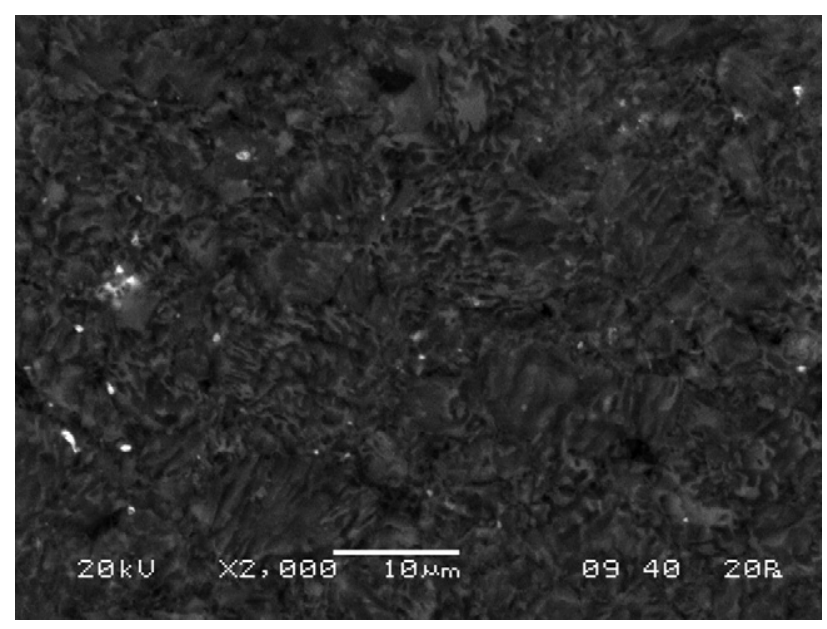

Figure 6. SEM image of a sample of PS 2450 prepared with dithranol and AgTFA. This image is higher magnification $(2000 \times)$ of a smooth region in Figure 5. Even at 2000x, we observe a very smooth, continuous film. It is difficult to quantitatively measure the typical dithranol particle size from these images. 

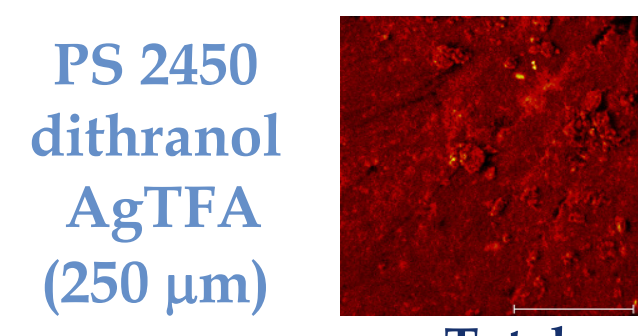

Total
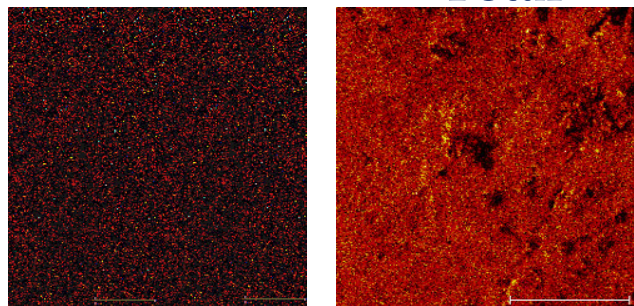

PS - polymer PS - Fragment

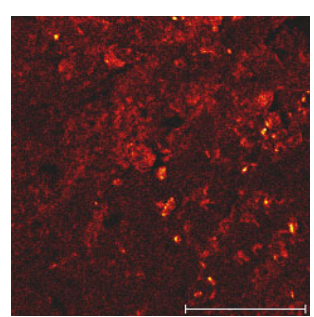

dithranol

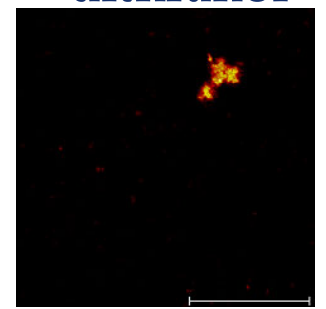

$\mathrm{Na}$

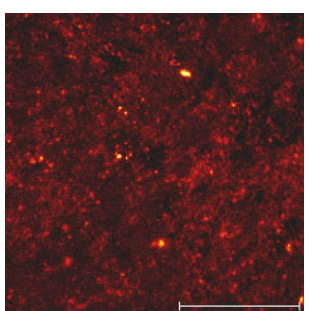

Ag

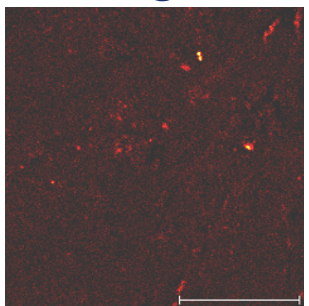

Al

Figure 7. ToF-SIMS ion specific images obtained from a sample of PS 2450 prepared with dithranol and AgTFA taken with a low lateral resolution $(250 \times 250 \mu \mathrm{m}$ raster). These data correlate well with the SEM data showing a homogeneous plain containing polymer, matrix and salt, and boulders containing matrix and salt.

thought these ions represented small pinholes in the film, but these samples also showed many charging issues, even with the charge compensation operating. Another source of these $\mathrm{Al}^{+}$ions is that during the spatula application process, the stainless steel spatula scratches the $\mathrm{Al}$ target, creates some very small particles of $\mathrm{Al}$, and distributes them evenly in the sample. Since stainless steel is much harder than $\mathrm{Al}$, we would expect the spatula application to scratch the $\mathrm{Al}$ substrate. The charging observed in the SIMS experiment is not consistent with the $\mathrm{Al}$ ions coming from imperfections in the thin film. An additional experiment using a plastic spatula to transfer the sample from the vortexing vial to the MALDI target showed no significant $\mathrm{Al}$ ion intensity. These data support the idea that the observed $\mathrm{Al}$ ion images are the result of using the metal spatula during the sample preparation.

\section{Conclusions}

Using AFM, SEM, and SIMS images, we have demonstrated that the relatively simple vortex method for solvent-free sample preparation for MALDI creates remarkably homogeneous and complete thin film sam-
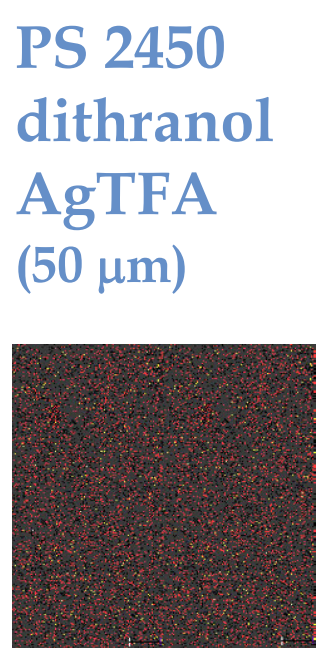

PS - polymer

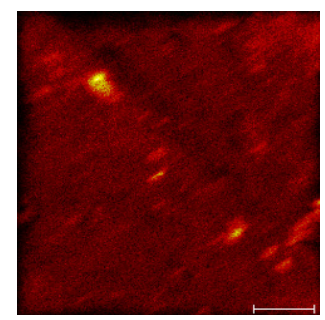

Total

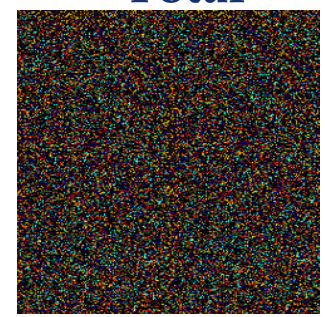

PS - Fragment

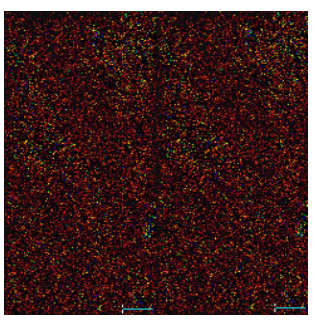

Dithranol

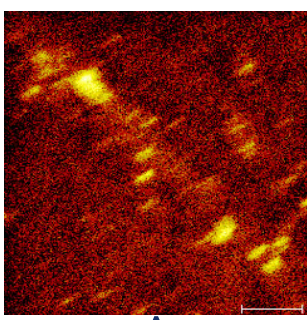

Ag

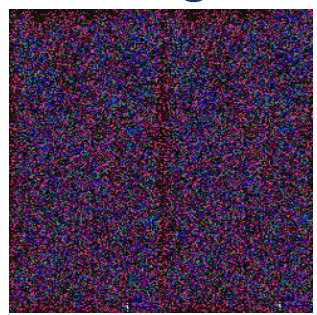

Al

Figure 8. ToF-SIMS ion specific images obtained from a sample of PS 2450 prepared with dithranol and AgTFA taken with a high lateral resolution $(50 \times 50 \mu \mathrm{m}$ raster) from a relatively smooth region of the sample. These data show a homogeneous plain containing polymer, matrix, and salt. 
ples. The images show that $60 \mathrm{~s}$ of vortexing fundamentally changes the size distribution and the shape of the matrix crystals. The SEM and the SIMS data show high levels of charging, indicating that these thin films have few flaws that might dissipate the charge. The individual ion images from the SIMS experiment show that the matrix, analyte, and cationization agent are evenly distributed throughout the images.

The homogeneous distributions in thin films helps to explain the high data quality obtained from the vortex prepared samples. How the simple vortex method distributes all of the components of the sample so quickly and evenly is still unknown.

\section{Acknowledgments}

We thank Air Products and Chemicals, Inc., for their support of this research, Prof. Kevin Owens and Bill Erb from Drexel University for helpful discussions about these experiments, Dr. Sarah Trimpin from Indiana University for helpful discussions of her solvent-free sample preparation technique, Drs. Paula Clark and Jiyun $\mathrm{Xu}$ for help running the ToF-SIMS experiments, Dr. Bob Pinschmidt and Khalil Yacoub for the PEF samples, and Dr. Sherri Bassner for critical review of the manuscript.

\section{References}

1. Tanaka, K.; Waki, H.; Ido, Y.; Akita, S.; Yoshido, Y.; Yoshido, T. Protein and Polymer Analyses up to m/z 100,000 by Laser Ionization Time-ofFlight Mass Spectrometry. Rapid Commun. Mass Spectrom. 1988, 2, 151-153.

2. Karas, M.; Hillenkamp, F. Laser Desorption Ionization of Proteins with Molecular Masses Exceeding 10,000 Daltons. Anal. Chem. 1988, 60, 2299-2301.

3. Bahr, U.; Deppe, A.; Karas, M.; Hillenkamp, F.; Giessman, U. Mass Spectrometry of Synthetic Polymers by UV-Matrix-Assisted Laser Desorption/Ionization. Anal. Chem. 1992, 64, 2866-2869.

4. Danis, P.; Karr, D.; Mayer, F.; Holle, A.; Watson, C. The Analysis of Water-Soluble Polymers by Matrix-Assisted Laser Desorption Time-ofFlight Mass Spectrometry. Org. Mass Spectrom. 1992, 27, 843-846.

5. Hanton, S. D. Mass Spectrometry of Polymers and Polymer Surfaces. Chem. Rev. 2001, 101, 527-569.

6. Nielen, M. W. F. MALDI Time-of-Flight Mass Spectrometry of Synthetic Polymers. Mass Spectrom. Rev. 1999, 18, 309-344.

7. Montaudo, G.; Lattimer, R. P., Eds. Mass Spectrometry of Polymers, CRC Press: Boca Raton, FL, 2002.

8. Pash, H.; Schrepp, W., Eds. MALDI-TOF Mass Spectrometry of Synthetic Polymers, Springer-Verlag: Berlin, 2003.

9. Wallace, W. E.; Guttman, C. M.; Hanton, S. D. Quantitative Synthetic Polymer Mass Spectrometry Workshop. J. Res. Natl. Inst. Stand. Technol. 2003, 108, 79-85.

10. Wallace, W. E.; Guttman, C. M.; Wetzel, S. J.; Hanton, S. D. Mass Spectrometry of Synthetic-Polymer Mixtures Workshop. Rapid Commun. Mass Spectrom. 2004, 18, 518-521.
11. Przybilla, L.; Brand, J.-D.; Yoshimura, K.; Räder, H. J.; Müllen, K. MALDI-TOF Mass Spectrometry of Insoluble Giant Polycyclic Aromatic Hydrocarbons by a New Method of Sample Preparation. Anal. Chem. 2000, 72, 4591-4597.

12. Trimpin, S.; Rouhanipour, A.; Räder, H. J.; Müllen, K. New Aspects in Matrix-Assisted Laser Desorption/Ionization Time-of-Flight Mass Spectrometry: A Universal Solvent-Free Sample Preparation. Rapid Commun. Mass Spectrom. 2001, 15, 1364-1373.

13. Gies, A. P.; Nonidez, W. K.; Anthamatten, M.; Cook, R. C.; Mays, J. W. Characterization of an Insoluble Polyimide Oligomer by MatrixAssisted Laser Desorption/Ionization Time-of-Flight Mass Spectrometry. Rapid Commun. Mass Spectrom. 2002, 16, 1903-1910.

14. Trimpin, S.; Keune, S.; Räder, H. J.; Müllen, K. Solvent-Free MALDI-MS: Developmental Improvements in the Reliability and the Potential of MALDI in the Analysis of Synthetic Polymers and Giant Organic Molecules. J. Am. Soc. Mass Spectrom. 2006, 17, 661-671.

15. Trimpin, S.; Deinzer, M. L. Solvent-Free MALDI-MS for the Analysis of a Membrane Protein via the Mini Ball Mill Approach: Case Study of Bacteriorhodopsin. Anal. Chem. 2007, 79, 71-78.

16. Hanton, S. D.; Parees, D. M. Extending the Solvent-Free MALDI Sample Preparation Method. J. Am. Soc. Mass Spectrom. 2005, 16, 90-93.

17. Trimpin, S.; Räder, H. J.; Müllen, K. Investigations of Theoretical Principles for MALDI-MS Derived from Solvent-Free Sample Preparation, Part I. Preorganization. Int. J. Mass Spectrom. 2006, 253, 13-21.

18. Horneffer, V.; Glückmann, M.; Krüger, R.; Karas, M.; Strupat, K.; Hillenkamp, F. Matrix-Analyte Interaction in MALDI-MS: Pellet and Nano-Electrospray Preparations. Int. J. Mass Spectrom. 2006, 249/250, $426-432$.

19. For an indepth overview of optical microscopy we refer the reader to the following website: http://micro.magnet.fsu.edu/primer/.

20. Newbury, D. E.; Joy, D. C.; Echlin, P.; Fiori, C. E.; Goldstein, J. I. Advanced Scanning Electron Microscopy and X-Ray Microanalysis, Plenum Press: New York, 1986.

21. Bonnell, D. A., Ed. Scanning Probe Microscopy and Spectroscopy: Theory, Techniques, and Applications, Wiley-VCH: New York, 2001.

22. Pacholski, M. L.; Winograd, N. Imaging with Mass Spectrometry. Chem. Rev. 1999, 99, 2977-3005.

23. Hanton, S. D.; Hyder, I. Z.; Stets, J. R.; Owens, K. G.; Blair, W. R.; Guttman, C. M.; Giuseppetti, A. A. Investigations of Electrospray Sample Deposition for Polymer MALDI Mass Spectrometry. J. Am. Soc. Mass Spectrom. 2004, 15, 168-179.

24. Hanton, S. D.; Cornelio Clark, P. A.; Owens, K. G. Investigations of Matrix-Assisted Laser Desorption/Ionization Sample Preparation by Time-of-Flight Secondary Ion Mass Spectrometry. J. Am. Soc. Mass Spectrom. 1999, 10, 104-111.

25. Hanton, S. D.; Owens, K. G.; Chavez-Eng, C.; Hoberg, A. M.; Derrick, P. J. Updating Evidence for Cationization of Polymers in the Gas Phase during Matrix-Assisted Laser Desorption/Ionization. Eur. J. Mass Spectrom. 2005, 11, 23-29.

26. In all of the imaging experiments (AFM, SEM, and ToF-SIMS) the general morphology results were observed to be independent of the polymer analyte or the salt cationization agent. All of the features of the observed morphology correlated with the matrix.

27. If we assume that the original CHCA particle is a sphere of radius $=250$ $\mu \mathrm{m}$, and the final CHCA particle after vortex mixing can be described as a cylinder with radius $=125 \mathrm{~nm}$ and a length of $1 \mu \mathrm{m}$, then the ratio of their volumes is $V_{s} / V_{c}=\left(4 / 3 \pi r_{s}^{3}\right) /\left(\pi r_{c}^{2} h\right)=4 r_{s}^{3} / 3 r_{c}^{2} h=4(250$ $\mu \mathrm{m})^{3} / 3(0.125 \mu \mathrm{m})^{2}(1 \mu \mathrm{m})=1.33 \times 10^{9}$, or about a billion times reduction in volume.

28. The AFM scanner's vertical scan range is limited to 6.5 microns. The peak-to-valley heights of the sample prepared with dithranol as the matrix was greater than 6.5 microns preventing us from scanning over the surface. Individual particles or particle aggregates could be contributing to this surface roughness. In the future we plan to examine methods to prepare smoother films with dithranol as the matrix. 